\title{
ORIGINAL ARTICLE \\ Rapid progressive clinical deterioration of cervical spondylotic myelopathy
}

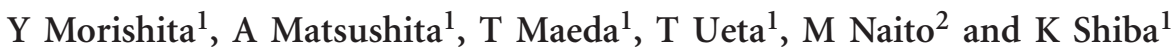

Study Design: Retrospective clinical study.

Objective: To elucidate the pathophysiology of rapid progressive clinical deterioration following the onset of cervical myelopathy. Setting: Spinal Injuries Center, Fukuoka, Japan.

Methods: A total of 43 cervical spondylotic myelopathy (CSM) patients were treated surgically by a senior surgeon. All patients showed intramedullary intensity changes on magnetic resonance (MR) imaging. Overall, eight patients suffered rapid progressive clinical deterioration; four of them had obvious anamnesis of minor trauma. We assessed the responsible injured segment by MR T2-weighted images. Clinical instabilities at the focal segment were evaluated using functional sagittal plain radiographs. Neurological evaluations were performed preoperatively and at 12 months postoperatively using American Spinal Injury Association (ASIA) motor scores and Japanese Orthopaedic Association (JOA) scores for cervical myelopathy. Intraoperatively, we evaluated the presence of adhesive scar tissue on the dura mater at the focal segment.

Results: The responsible injured segment was C3-4 in 75\% of the rapid progressive (rp)-CSM and in $28.57 \%$ of the conventional CSM subjects. One with rp-CSM showed sagittal translational segmental instability. Preoperative ASIA motor scores and JOA scores in the rp-CSM were significantly lower than those in the conventional CSM subjects. Postoperative ASIA motor scores between the subjects showed no significant differences; however, postoperative JOA scores in the rp-CSM subjects were significantly lower. Moreover, an epidural membrane was observed in $62.5 \%$ of rp-CSM and $11.4 \%$ of conventional CSM subjects.

Conclusions: We hypothesized that the pathophysiology of rp-CSM might be additional cervical cord disorder following the onset of cervical myelopathy. Early decompression surgery is recommended in such patients.

Spinal Cord (2015) 53, 408-412; doi:10.1038/sc.2014.137; published online 2 September 2014

\section{INTRODUCTION}

The etiologies of cervical spondylotic myelopathy (CSM) include spinal cord compression, dynamic factors and ischemia of the spinal cord. Several anatomical structures in the cervical motion segment can be involved in cervical spinal cord compression. The spondylotic changes in anterior structures, such as bulging, ossified or herniated discs, as well as anterior osteophytic spurs are generally responsible for cord compression in CSM. Disorders in posterior structures, such as hypertrophy, or rarely, ossification/calcification of the ligamentum flavum (CLF) or facet joints may also contribute to cord compression. Compression of the spinal cord within a narrow spinal canal is believed to cause neural injury and ischemia. The cord and its vascular supply have the ability to adapt to a chronic, slowly progressing compression; therefore, dynamic compression may be more important than static compression in the development of myelopathy symptoms. Patients with canal stenosis also have limited cord excursion, and spinal column motion may increase the strain and shear forces on the spinal cord. ${ }^{1}$

CSM is a relatively slowly progressive disorder. However, we rarely encounter CSM patients with rapidly progressive clinical deterioration following the onset of cervical myelopathy in the clinical setting. We assumed that there might be certain differences in the pathophysiology between conventional cervical myelopathy and rapid progressive clinical deterioration following the onset of cervical myelopathy.
CSM is a disorder that is typically treated surgically. Early treatment before the onset of permanent cord injury is recommended. ${ }^{2}$ Therefore, it is important for physicians to recognize the pathophysiology of CSM. Numerous studies have reported the pathogenesis of CSM. However, to the best of our knowledge, few reports have thus far referred to the state of rapid progressive clinical deterioration following the onset of cervical myelopathy. The purpose of this retrospective clinical study was to elucidate the pathophysiology of rapid progressive clinical deterioration of CSM.

\section{MATERIALS AND METHODS}

\section{Study population}

Between January 2010 and December 2012, a total of 43 CSM patients (27 men and 16 women; average age, 70.9 years; range, 44-92 years) were treated surgically by a senior spine surgeon. All patients showed cervical cord intramedullary intensity changes on magnetic resonance (MR) T2-weighted images (Figure 1a), and demonstrated long tract signs, such as fine motion disturbances in the fingers, gait disturbance and deep tendon hyperreflexia in the lower limbs. Regarding clinical manifestations, 35 CSM patients presented with cervical spondylosis, while 8 presented with cervical ossification of the posterior longitudinal ligament (OPLL). CSM patients who were treated with anterior decompression and fusion or who had a history of any spinal surgery before or after the cervical surgery were excluded from the study.

Overall, 8 of 43 CSM patients ( 4 males and 4 females), including 1 patient with cervical OPLL, with an average age of 73.5 years suffered rapid progressive

${ }^{1}$ Department of Orthopedic Surgery, Spinal Injuries Center, lizuka, Japan and 2Department of Orthopedic Surgery, Fukuoka University, Fukuoka, Japan

Correspondence: Dr Y Morishita, Department of Orthopedic Surgery, Spinal Injuries Center, 550-4, Igisu, lizuka 820-0053, Japan.

E-mail: uchiro1968@mac.com

Received 1 April 2014; revised 29 May 2014; accepted 8 July 2014; published online 2 September 2014 

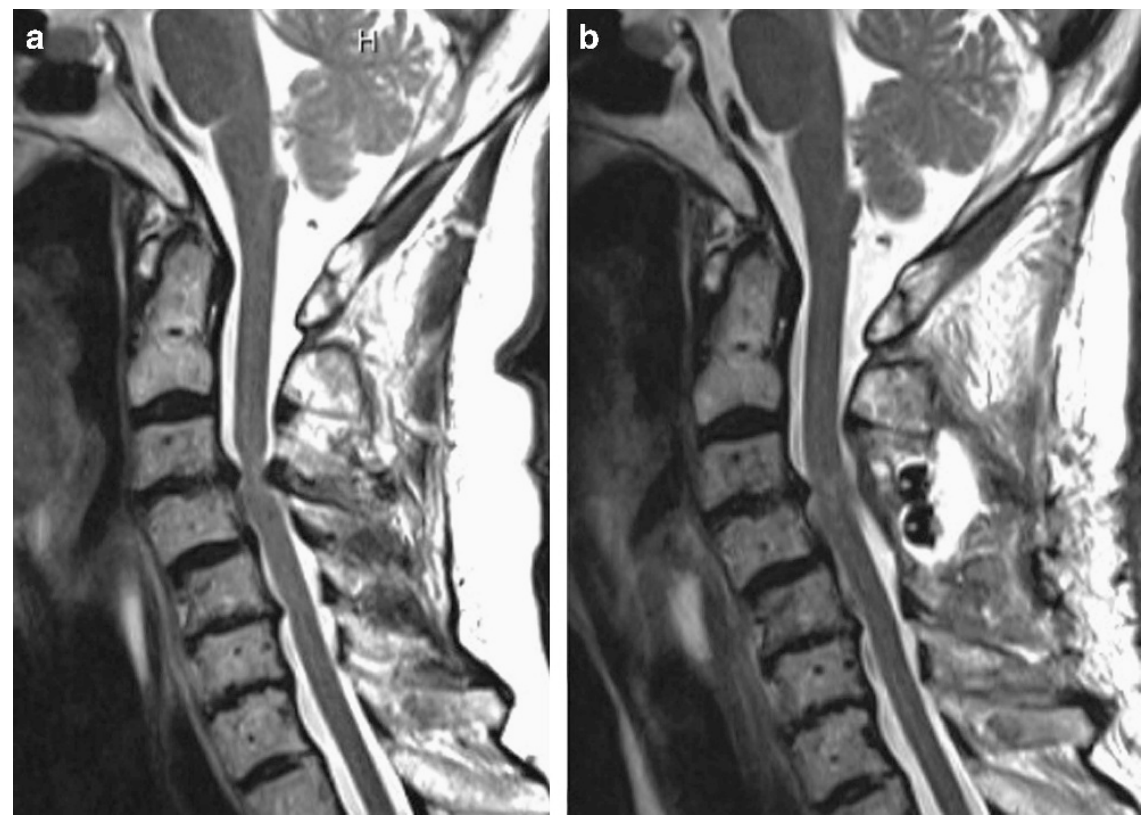

Figure 1 Intramedullary cervical cord intensity changes on MR T2-weighted images at the C3-4 segment. (a) Preoperative image. (b) Postoperative image.

clinical deterioration (rp-CSM); none of these patients could maintain standing posture without support within 4 weeks of the onset of symptoms because of deterioration of the existing cervical myelopathy. Of these, four subjects had obvious anamnesis of minor trauma, such as stumbling over or minor falls, before clinical deterioration. Thus, 35 of 43 CSM patients (23 males and 12 females), including 7 patients with cervical OPLL, with an average age of 70.3 years demonstrated conventional cervical myelopathy (CSM).

The study was approved by our Institutional Review Board, and informed consent was obtained from all the patients.

\section{Radiographic analysis}

We assessed the responsible injured segment by examining intramedullary intensity changes on MR T2-weighted images. The clinical instabilities at the focal cervical segment were evaluated using functional sagittal plain radiographs as defined by White et al. ${ }^{3}$ (more than $3.5 \mathrm{~mm}$ of dynamic translation or $11^{\circ}$ greater angulation than that in the adjacent segment) (Figure 2).

\section{Neurological evaluations}

The clinical assessments (upper and lower limb functions) were performed preoperatively and at 12 months postoperatively on the basis of American Spinal Injury Association (ASIA) motor scores (upper limb: range, 0-50; lower limb: range, 0-50; total: range, $0-100)^{4}$ and Japanese Orthopaedic Association (JOA) scores for the evaluation of cervical myelopathy (upper limb: range, 0-6; lower limb: range, $0-6$; total: range, $0-17) .^{5}$

\section{Surgical treatment: intraoperative findings}

All of the patients underwent French-door cervical laminoplasty. ${ }^{6}$ Using the threadwire saw to perform the sagittal split in the spinous process, the hydroxyapatite blocks were placed as a strut to maintain the patency of the hinged hemilaminae (Figure 3$)^{7}$

Intraoperatively, we evaluated each patient for the presence of CLF or adhesive scar tissue on the dura mater (epidural membrane) at the focal segment.

\section{Statistical analysis}

The Mann-Whitney $U$ test was used for all statistical analyses. $P<0.05$ was considered statistically significant.

\section{RESULTS}

There was no significant difference in age between the rp-CSM and CSM subjects. Moreover, gender and the presence of cervical OPLL did not appear to affect the condition of cervical myelopathy.

\section{Radiographic analysis}

Among the rp-CSM subjects, the responsible injured segment was C34 in six patients (75\%); C4-5 in one patient (12.5\%); and C5-6 in one patient $(12.5 \%)$. Among the CSM subjects, the responsible injured segment was C3-4 in 10 patients (28.57\%); C4-5 in 15 patients (42.86\%); and C5-6 in 10 patients (28.57\%). Only one subject with rp-CSM showed sagittal dynamic translational instability on the functional plain radiographs $(3.75 \mathrm{~mm}$ of dynamic translational instability) (Figure 2). Two CSM subjects demonstrated more than $3.5 \mathrm{~mm}$ (5.35 and $6.9 \mathrm{~mm}$ ) of translational anterior slip on flexion radiographs; however, these subjects showed less than $3.5 \mathrm{~mm}$ of dynamic translational instability.

\section{Neurological evaluations}

Table 1 shows the ASIA motor scores for rp-CSM and CSM subjects examined preoperatively and at 12 months postoperatively. All of the scores for the rp-CSM and CSM subjects, except the lower limb score for CSM, significantly improved at 12 months postoperatively. The preoperative scores, including the upper limb, lower limb and total scores, for rp-CSM subjects were significantly lower than those for CSM subjects; however, no significant differences were observed in the scores at 12 months postoperatively between the subjects. Table 2 shows the JOA scores and JOA score recovery ratio for rp-CSM and CSM subjects examined preoperatively and at 12 months postoperatively. All of the scores for rp-CSM and CSM subjects significantly improved at 12 months postoperatively. However, all the preoperative and postoperative scores for rp-CSM subjects were significantly lower than the corresponding scores for CSM subjects.

\section{Intraoperative findings}

Overall, 3 of 35 CSM subjects but none of the rp-CSM subjects demonstrated CLF at the focal segment. However, intraoperative 


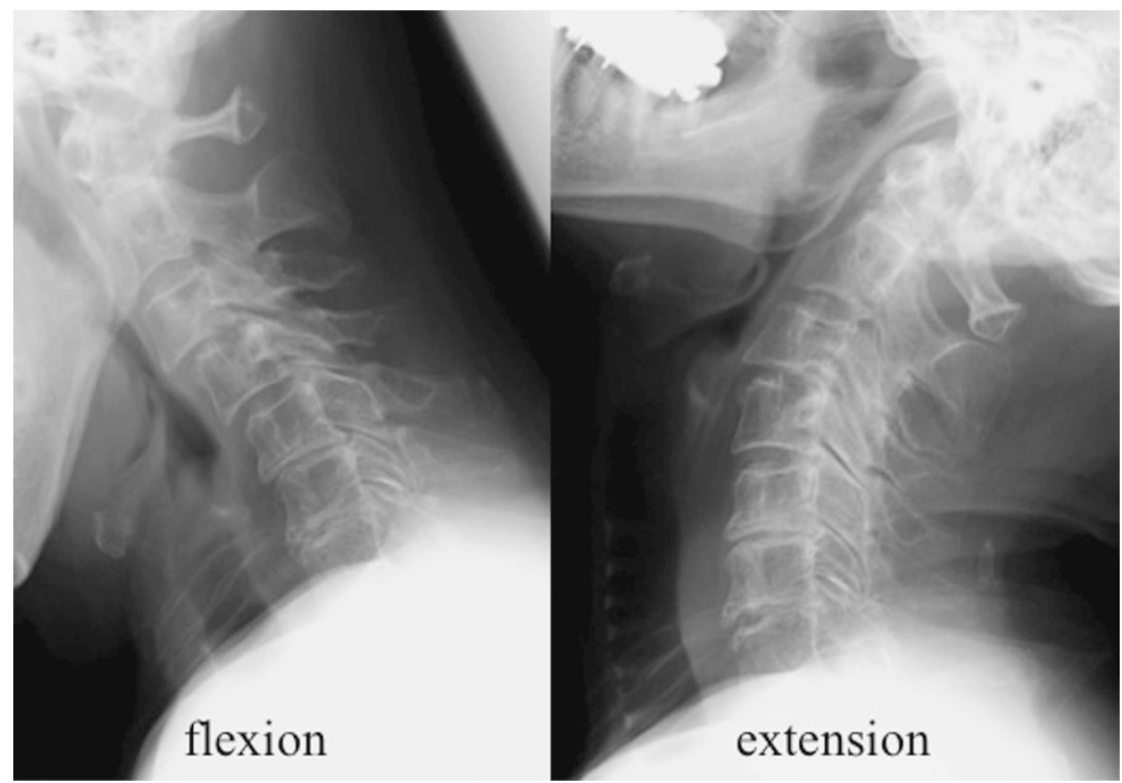

Figure 2 Functional sagittal plain radiographs with flexion and extension images. The images showed $3.75 \mathrm{~mm}$ of dynamic translational instability at the C3-4 segment.

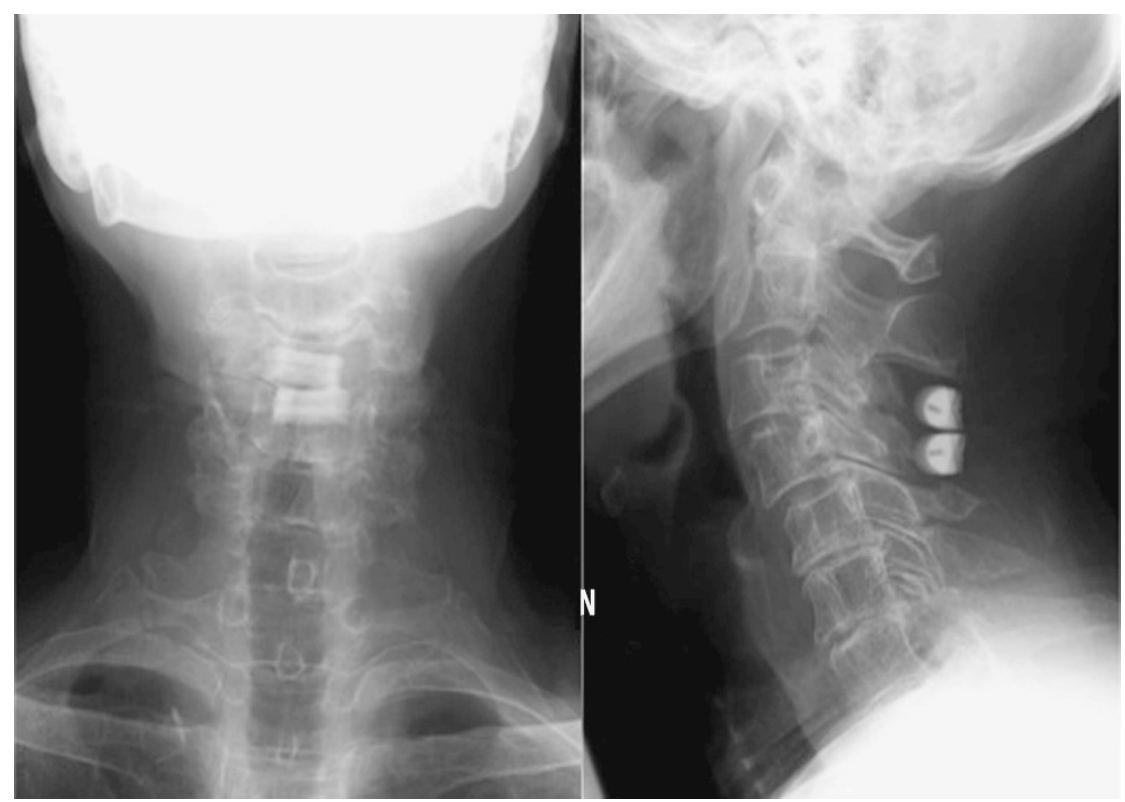

Figure 3 French-door laminoplasty using hydroxyapatite blocks at the C3 and C4 laminar surface: postoperative image.

Table 1 ASIA motor scores

\begin{tabular}{|c|c|c|c|c|c|c|}
\hline & \multicolumn{3}{|c|}{ Preoperative } & \multicolumn{3}{|c|}{ Postoperative } \\
\hline & Upper & Lower & Total & Upper & Lower & Total \\
\hline \multirow[t]{2}{*}{ rp-CSM } & $35.2 \pm 2.4$ & $41.8 \pm 2.3$ & $77 \pm 4.5$ & $45 \pm 2.8^{* * *}$ & $48.8 \pm 1.1^{* * *}$ & $93.8 \pm 2.9^{* * *}$ \\
\hline & $\dagger \dagger$ & $\dagger \dagger$ & $\dagger \dagger$ & ns & ns & ns \\
\hline CSM & $43.3 \pm 5.2$ & $48.5 \pm 3.3$ & $91.8 \pm 6.4$ & $46.8 \pm 3.6^{*}$ & $49 \pm 2.7$ & $95.8 \pm 4.7^{*}$ \\
\hline
\end{tabular}

Abbreviations: ASIA, American Spinal Injury Association; CSM, cervical spondylotic myelopathy; ns, not significant; rp-CSM, rapid progressive cervical spondylotic myelopathy. Comparison between preoperative and postoperative scores: ${ }^{*} P<0.05,{ }^{* *} P<0.01,{ }^{* * *} P<0.001$ Comparison between rp-CSM and CSM patients: $\uparrow P<0.05, \uparrow \dagger P<0.01, \dagger \dagger \uparrow P<0.001$ 
Table 2 JOA scores for cervical myelopathy

\begin{tabular}{|c|c|c|c|c|c|c|}
\hline & \multicolumn{3}{|c|}{ Preoperative } & \multicolumn{3}{|c|}{ Postoperative } \\
\hline & Upper & Lower & Total & Upper & Lower & Total \\
\hline rp-CSM & $+\dagger \dagger$ & $\dagger \dagger$ & $\dagger \dagger$ & $\dagger$ & $\dagger$ & $\dagger$ \\
\hline CSM & $2.4 \pm 0.7$ & $3.5 \pm 1.2$ & $10.1 \pm 1.8$ & $3.7 \pm 0.6^{* * *}$ & $4.4 \pm 1.3^{*}$ & $12.6 \pm 1.9^{* *}$ \\
\hline
\end{tabular}

Abbreviations: CSM, cervical spondylotic myelopathy; JOA, Japanese Orthopaedic Association; rp-CSM, rapid progressive cervical spondylotic myelopathy.

Comparison between preoperative and postoperative scores: ${ }^{*} P<0.05,{ }^{* *} P<0.01,{ }^{* * *} P<0.001$.

Comparison between rp-CSM and CSM patients: $\dagger P<0.05, \dagger \dagger P<0.01, \dagger \dagger \uparrow P<0.001$.
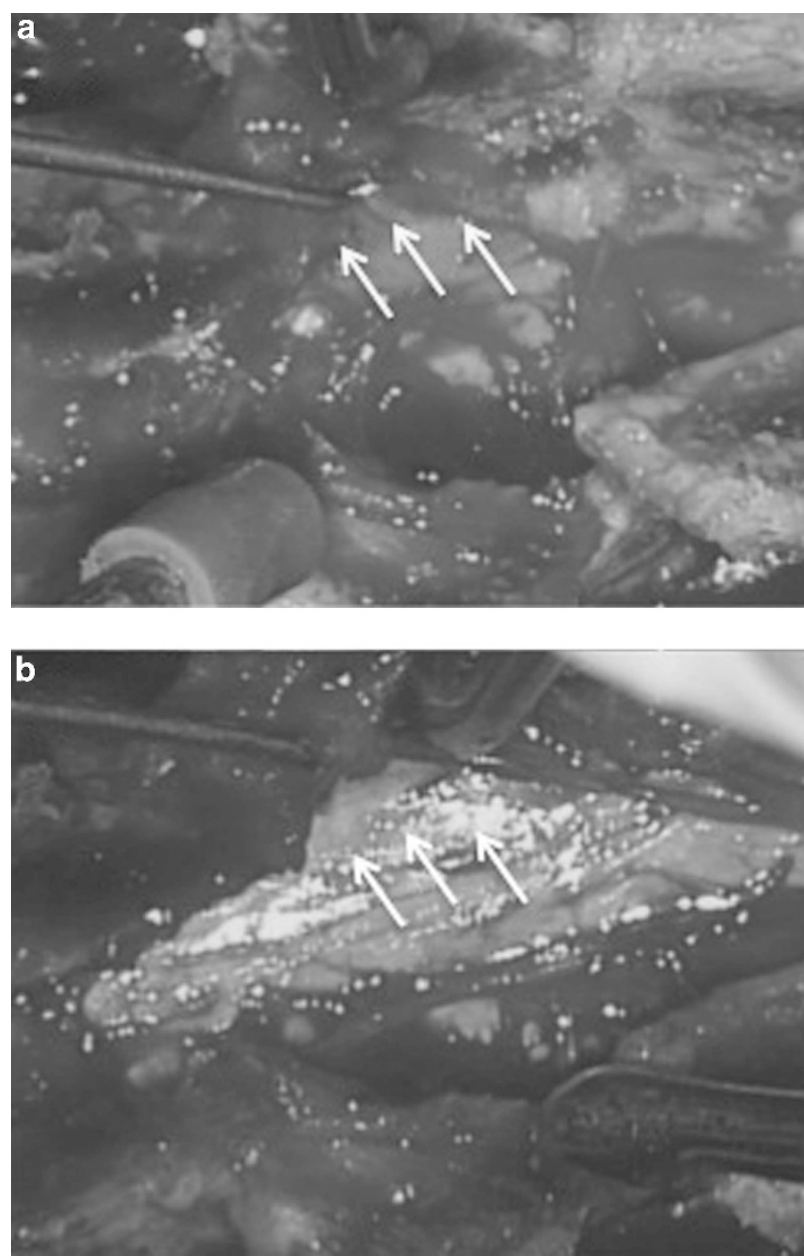

Figure 4 Intraoperative findings. (a) Adhesive scar tissue on the dura mater at the focal segment. (b) After adhesiotomy.

findings revealed adhesive scar tissue on the dura mater (epidural membrane) at the focal segment in 5 of $8(62.5 \%)$ rp-CSM subjects and 4 of 35 (11.4\%) CSM subjects (Figure 4$)$.

\section{DISCUSSION}

CSM is the development of long tract signs secondary to degenerative changes in the cervical spinal column. MR imaging (MRI) can provide various diagnostic information and has become essential as the gold standard in diagnosing CSM. ${ }^{8}$ In particular, the existence of intramedullary intensity changes as well as the effacement of the subarachnoid space and spinal cord compression on MRI helps us to diagnose patients with CSM. ${ }^{9}$ Intramedullary intensity changes on MRI have been reported to reflect irreversible changes in the spinal cord, to be related to pyramidal signs, and to be indicative of the prognosis, although this remains controversial. ${ }^{10-13}$ However, the extent of spinal cord compression, even with intramedullary intensity changes on MRI, does not necessarily correlate with that of clinical symptoms.

The natural history of cervical myelopathy most frequently involves a stepwise deterioration in neurological function, with stable plateau periods. Oshima et al. ${ }^{14}$ reported that $82 \%$ and $56 \%$ of CSM patients were still conservatively followed without serious neurological deterioration at 5 and 10 years after the initial treatment, respectively. They also reported that large cervical ROM, segmental kyphosis or presence of a slip in the maximum compression segment significantly affected the prognosis. Although there are several possible courses of development for CSM, including the stabilization of symptoms, this disease often presents with progressive clinical deterioration and may lead to severe disability. ${ }^{15}$

In our series, eight CSM subjects demonstrated rapid progressive clinical deterioration within 4 weeks of symptomatic onset, and four of eight subjects suffered clinical deterioration with obvious minor trauma as a trigger. On the basis of our results, we consider that the age, gender or existing OPLL at the focal segment might not affect the development of neurological deterioration. However, the responsible injured segment in cervical myelopathy being C3-4 might predispose the patient to rapid progressive clinical deterioration.

Dynamic instability at the focal segment was observed in one of the eight rp-CSM patients and in none of the conventional CSM patients. We consider that the clinical instabilities at the focal segment might be an important factor in the progressive clinical deterioration of cervical myelopathy. In such patients, the cervical cord might be exposed to major dynamic stresses in addition to static compression.

Preoperative clinical assessments using ASIA motor and JOA scores for the rp-CSM subjects were significantly lower than those for the conventional CSM subjects. However, all of the scores, except the lower limb score for conventional CSM evaluated with ASIA motor scores, significantly improved at 12 months postoperatively. Moreover, all of the postoperative ASIA motor scores, which reflect the limbs' motor functions, showed no significant differences between rpCSM and conventional CSM subjects. High positive postoperative reaction to surgical decompression, such as adhesiotomy and laminoplasty, was observed not only for the conventional CSM subjects but also for the rp-CSM subjects. With respect to JOA scores, which reflect the activity of daily living (ADL) functions, although significant improvements were observed in each score for both rp-CSM and conventional CSM subjects at 12 months postoperatively, the postoperative scores in the rp-CSM subjects were significantly lower than those in the conventional CSM subjects. 
These results suggested that surgical treatment might induce improvements in limb motor function; however, cervical myelopathy symptoms that affect the patient's ADL may remain severe for rp-CSM subjects. Any additional cervical cord disorder by minor trauma to the cervical cord with existing cervical myelopathy might lead to rapid progressive neurological deterioration. From our results, early decompressive surgical treatment is recommended in patients with rapid progressive clinical deterioration following the onset of cervical myelopathy.

Several studies have reported that a pathological epidural membrane is located at approximately the most damaged spinal segment in CSM, which may reflect greater degenerative changes in the neural tissue and its surroundings under continuous static and dynamic compression at this segment. ${ }^{16-18}$ Miyauchi et al. ${ }^{16}$ hypothesized that during the formation of such a pathological epidural membrane at the most damaged segment, active metabolism may occur because of repetitive tissue damage and repair under continuous mechanical stresses at this segment. In our series, the evidence of CLF at the focal segment did not involve the development of neurological deterioration of cervical myelopathy. However, the presence of an epidural membrane at the focal segment was observed in $62.5 \%$ of the rp-CSM subjects. Histopathological examination of tissue sections revealed fibro-collagenous tissue without atypical cells or inflammatory infiltration. We also hypothesized that repetitive tissue damage and repair or occasional bleeding from the epidural venous plexus following continuous mechanical stresses at the focal segment may lead to the formation of adhesive scar tissue on the dura mater at the focal segment. Additional dynamic stresses on the entrapped cervical spinal cord with the presence of the epidural membrane may play an important role in the development of rapid progressive clinical deterioration of cervical myelopathy. The pathophysiology of rapid progressive clinical deterioration of CSM might thus be additional cervical cord disorder on a cervical cord with existing cervical myelopathy.

Certain issues remain unaddressed in the current study. We have only discussed mid-term surgical outcomes. Early recovery of motor functions, including those of the upper and lower limbs, evaluated using ASIA motor scores were observed in the rp-CSM subjects. However, with respect to JOA scores for cervical myelopathy that reflect ADL functions, the rp-CSM subjects demonstrated significantly lower scores as compared with corresponding scores for the conventional CSM subjects. The natural course of ADL functional recovery in the long term in CSM patients remains unclear. Using the current investigation as a pilot study, further research using a larger patient population and long-term follow-up may help in resolving several remaining unclear issues. Moreover, the pathophysiology of rapid progressive clinical deterioration following the onset of cervical myelopathy needs to be clarified in greater detail.

\section{DATA ARCHIVING}

There were no data to deposit.

\section{CONFLICT OF INTEREST}

The authors declare no conflict of interest.

1 Kalantar SB, Yoon ST. The pathophysiology of cervical spondylotic myelopathy. In: Rao RD and Smuck M (eds). Orthopaedic Knowledge Update: Spine 4, 4th edn, vol. 5. AAOS: Rosemont, IL, 2012, pp 51-59.

2 Schmitt-Sody M, Kirchhoff C, Buhmann S, Metz P, Birkenmaier C, Troullier H et al. Timing of cervical spine stabilization and outcome in patients with rheumatoid arthritis. Int Orthop 2008; 32: 511-516.

3 White AA III, Johnson RM, Panjabi MM, Southwick WO. Biomechanical analysis of clinical stability in the cervical spine. Clin Orthop Relat Res 1975; 109: 85-96.

4 American College of Surgeons. American College of Surgeons Committee on Trauma. Advanced Trauma Life Support: ATLS. 7th ed. Chicago 2004.

5 Hirabayashi K, Watanabe K, Wakano K, Suzuki N, Satomi K, Ishii Y. Expansive opendoor laminoplasty for cervical spinal stenotic myelopathy. Spine (Phila Pa 1976) 1983; 8: 693-699.

6 Kurokawa T, Tsuyama N, Tanaka H. Enlargement of spinal canal by the sagittal splitting of the spinous process. Bessatsu Seikei Geka 1982; 2: 234-240.

7 Tomita K, Kawahara N, Toribatake Y, Heller JG. Expansive midline T-saw laminoplasty (modified spinous process-splitting) for the management of cervical myelopathy. Spine (Phila Pa 1976) 1998; 23: 32-37.

8 Al-Mefty O, Harkey LH, Middleton TH, Smith RR, Fox JL. Myelopathic cervica spondylotic lesions demonstrated by magnetic resonance imaging. J Neurosurg 1988; 68: 217-222.

9 Bednarik J, Kadanka Z, Dusek L, Kerkovsky M, Vohanka S, Novotny 0 et al. Presymptomatic spondylotic cervical myelopathy: an updated predictive model. Euro Spine J 2008; 17: 421-431.

10 Takahashi M, Yamashita Y, Sakamoto Y, Kojima R. Chronic cervical cord compression: clinical significance of increased signal intensity on MR images. Radiology 1989; 173 219-224.

11 Rhee JM, Helfin JA, Hamasaki T, Freedman B. Prevalence of physical signs in cervical myelopathy: a prospective, controlled study. Spine (Phila Pa 1979) 2009; 34: 890-895.

12 Yukawa $\mathrm{Y}$, Kato F, Ito K, Horie Y, Hida T, Machino M et al. Postoperative changes in spinal cord signal intensity in patients with cervical compression myelopathy: comparison between preoperative and postoperative magnetic resonance images J Neurosurg Spine 2008; 8: 524-528.

13 Mastronardi L, Elsawaf A, Roperto R, Bozzao A, Caroli M, Ferrante M et al. Prognostic relevance of the postoperative evolution of intramedullary spinal cord changes in signa intensity on magnetic resonance imaging after anterior decompression for cervical spondylotic myelopathy. J Neurosurg Spine 2007; 7: 615-622.

14 Oshima Y, Seichi A, Takeshita K, Chikuda H, Ono T, Baba S et al. Natural course and prognostic factors in patients with mild cervical spondylotic myelopathy with increased signal intensity on T2-weighted magnetic resonance imaging. Spine (Phila Pa 1979) 2012: 37: 1909-1913.

15 Clarke E, Robinson P. Cervical myelopathy: a complication of cervical spondylosis. Brain 1956; 95: 87-100.

16 Miyauchi A, Sumida T, Manabe H, Mikami Y, Kaneko M, Sumen Y et al. Morphological features and clinical significance of epidural membrane in the cervical spine. Spine (Phila Pa 1979) 2012; 37: E1182-E1188.

17 Seichi A, Takeshita K, Kawaguchi H, Matsudaira K, Higashikawa A, Ogata N et al. Neurologic level diagnosis of cervical stenotic myelopathy. Spine (Phila Pa 1979) 2006; 31: 1338-1343.

18 Matsumoto M, Ishikawa M, Ishii K, Nishizawa T, Maruiwa H, Nakamura M et al. Usefulness of neurological examination for diagnosis of the affected level in patients with cervical compressive myelopathy: prospective comparative study with radiological evaluation. J Neurosurg Spine 2005; 2: 535-539. 